\title{
A review on data clustering using spiking neural network (SNN) models
}

\author{
Siti Aisyah Mohamed, Muhaini Othman, Mohd Hafizul Afifi \\ Faculty of Computer Science and Information Technology, University Tun Hussein Onn, Malaysia
}

\begin{tabular}{l} 
Article Info \\
\hline Article history: \\
Received Dec 2, 2018 \\
Revised Mar 17, 2019 \\
Accepted Apr 25, 2019 \\
\hline
\end{tabular}

\section{Keywords:}

Clustering

Deep learning

Machine learning

Spiking neural network

Temporal data

\begin{abstract}
The evolution of Artificial Neural Network recently gives researchers an interest to explore deep learning evolved by Spiking Neural Network clustering methods. Spiking Neural Network (SNN) models captured neuronal behaviour more precisely than a traditional neural network as it contains the theory of time into their functioning model [1]. The aim of this paper is to reviewed studies that are related to clustering problems employing Spiking Neural Networks models. Even though there are many algorithms used to solve clustering problems, most of the methods are only suitable for static data and fixed windows of time series. Hence, there is a need to analyse complex data type, the potential for improvement is encouraged. Therefore, this paper summarized the significant result obtains by implying SNN models in different clustering approach. Thus, the findings of this paper could demonstrate the purpose of clustering method using SNN for the fellow researchers from various disciplines to discover and understand complex data.
\end{abstract}

\section{Corresponding Author:}

Siti Aisyah Mohamed,

Faculty of Computer Science and Information Technology,

University Tun Hussein Onn, Malaysia.

Email: sitiaisyahmohamed@gmail.com

\section{INTRODUCTION}

Deep learning has become the new revolution in the arena of machine learning purposely for computer visualization. A deep (multilayer) artificial neural network (ANN) is taught in a controlled manner via back propagation and has been used vastly as computational tools to unravel difficult classification, regression, pattern recognition and function estimation problem as well as complicated optimization problem with highly non-linear and discontinuous constraints [2]. As a result of extensive studies in ANNs, has led to innovation of more advance type of networks such as self-organizing maps, recurrent networks, probabilistic neural networks, dynamic neural networks, radial basis function neural networks, complex valued neural networks, feed forward neural networks, genetic algorithm, fuzzy logic and wavelets . The most common technique applied to numerous instruments for a forward allocation of info through the neural network is feed forward ANNs because of the fact that method of information transmission in real and artificial neural networks is feed forward in nature equally and its simplicity [3]. However, a few drawbacks of ANNs are acknowledged as total of remarkable challenges and limitations [4]. Data multidimensional is among the notable limitations in supervised learning which happens when the amount of features and training points becomes ominously huge. Due to the resultant memory and computational necessities and the irresistible volume of data to practice makes ANN learning more challenging. Additional challenge in classification is characteristic feature overlap between different classes because the data is non-linear has made the chore of splitting the classes challenging. Focusing to improve on these drawbacks by utilizing heuristic method, Spiking Neural Network (SNNs) models of data clustering is reviewed in this paper. 


\section{CLUSTERING OVERVIEW}

Classification and clustering are both fundamental chores in data mining knowledge discovery. Classification techniques are used frequently in an administered learning method (supervised) while clustering techniques usually for unverified (unsupervised) learning in which the objective of clustering is descriptive; on the other hand classification is predictive [5]. To recognize the organizations in diverse data natures such as static, temporal and spatial temporal, many clustering method have been developed. Once the features values do not modify in excess of time, the static data type is identified whereas temporal data is considered as a series of time when the features change their value over an uninterrupted time. This is the classification criteria of clustering [6]: (a) Straight clustering of data against clustering of a model that is being learn and capture the necessary characteristic of concern from the data. (b) Clustering raw data against clustering of pre-processing data. (c) Clustering of dynamic, streaming data against vector based (static data) and (d) Clustering of complete values variable against clustering of fluctuations in the variable.

Fundamentally, there are five major categories of clustering method for various static data (vector data): (a) Hierarchical approaches build the clusters by recursively separating the occurrences either a topdown or bottom-up manner [7]. (b) Partitioning method which assigns the centre of data points as the centre of the corresponding cluster. (c) Density-based methods separate the object based on the connectivity, boundary and density as calculate in the neighbourhood nearest point [8]. (d) Grid based clustering quantized the object planetary into a limited amount of cells, wherever the clustering processes is accomplished [6]. (e) Model based clustering targets at the finest fit data to the model by undertake a model for each cluster [9]. This method is one of the major approaches of Neural Network clustering.

Corresponding with the advanced technology of computer science, the massive amount of data is produced. The data produced does not limit only to static data type but temporal data type is also being recorded by various areas such as biology, electronic, environment and also finance. This massive raw data recorded need a powerful analyzing tool to extract the information. Thus, cluster work for this type of data is also being introduced such as bi-clustering [10] that has been explored to cluster the time series gene analysis. This method capable to instantaneously clusters columns and row of a grid matrix together.

The objective of this bi-clustering methods is to cluster analogous pattern into the identical group where it able to cope with the temporal contact among the time series data feature [10]. However, this method is still not capable to handle the relationship between spatio data type and temporal data type. The dynamic association of temporal features plays an important role that influence the training and transformation of pattern dynamically overtime. Thus, spatio-temporal clustering technique has been recommended in order to enhance the capability of clustering approach to deal with static based data and spatio-temporal information simultaneously based on Spiking Neural Network modelling [6].

\section{CLUSTERING IN SPIKING NEURAL NETWORK}

Motivated by the utility and structure of how the human brain learn information where neuron models interconnect by structures of spikes, Spiking Neural Networks is presented. Networks collected from spiking neurons are capable to practice large volume of data with a moderately small amount of spikes [11]. Spiking networks works similarly to biological neurons can be seen in the dendrites, cell body and axon parts [12] as in Figure 1 which gives it the ability to solve a wide variety of definite problem in everyday engineering such as motor controller, event discovery, signal-processing, spatial navigating or speech recognition.

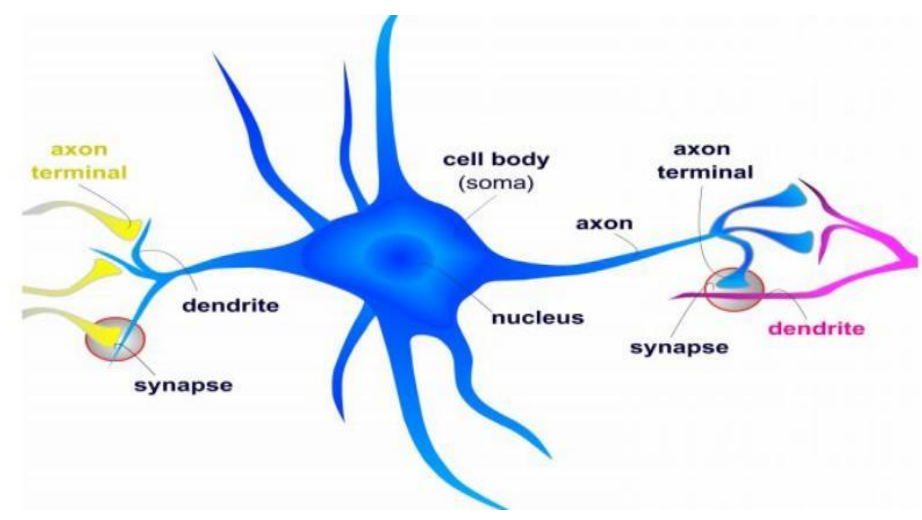

Figure 1. Biological neuron model taken from [13] 
A presynaptic neuron interconnects with a post-synaptic neuron via trains of spikes or stroke potentials. The transferred material is commonly programmed in the rate of recurrence of spiking (rate encoding) and the scheduling of the spikes (pulse encoding) [14]. Pulse encoding is extra influential than rate encoding in relations of the wide-ranging of info that may perhaps encoded by the identical number of neurons. Indeed, rate encoding can be reflected to be an exceptional instance of pulse encoding.

The average firing rate can be figured if the spike timings are identified. Nonetheless, the spiking neuron has a natural dynamic nature that is categorized by an interior state which varies upon time. Every post-synaptic, neuron fires an action possible or spike at the time occurrence its core state surpasses the neuron threshold [14]. Comparable to biological neurons, the weight of the spikes does not disclose any info.

In this paper, few applications of SNN in data clustering is presented. As an unsupervised learning, clustering tends to organize data samples into it homogeneous groups in which all the data samples within the group are similar constructed on particular designated possessions of the data [15]. Unsupervised clustering removes the requirement for several repetitions over a training data set which is typically obligatory for supervised learning procedures for example gradient lineage and its deviations [16] thus it require lower computational burden. Hence, many unsupervised learning applied SNNs as approached and its former development [3].

Thru adjusting synaptic strong point of Hebbian progressions lead to the redeployment of networks contained by a neural network and beneath certain circumstances might affect in the advent of innovative functions for instance input clustering, source separation, pattern recognition, dimensionality reduction, construction of self-organizing maps or construction of associative memories [11]. This practice usually implicates learning designed configurations in the data by eliminating untainted formless noise. Dimensionality reduction and clustering algorithms are typically unsupervised learning [17].

\section{CLUSTERING EVALUATION}

All the clustering approach used for different clustering application such as image segmentation, bio-informatics of object recognition, business, gene expression data, character recognition, data mining, information retrieval, spatial data analysis, data reduction and big data mining has to undergo a few evaluation criteria to test the legitimacy and accurateness of the clusters models performed. Good clustering algorithms or models are considered when the models are able to handle the dimensional criterion possession of the data which can recognize as 3V's concept. The concept are: (1) Volume - ability to deal with huge amount of data, (2) variety- capability of the clustering algorithms to grip different types of data or formats and (3) Velocity - The speed of the clustering algorithms on big data which are generated at high speed. The clusters molded by a certain scheme must be verified whether it display concentrated resemblance among the objects in the same cluster or slightest resemblance among those in other clusters [18]. The evaluation criterion is allocated into two kinds as internal quality criteria measures such as present in Table 1 and external quality criteria measures such as present in Table 2.

Table 1. Internal Quality Criteria Measure with Their Formula

\begin{tabular}{cc}
\hline Types & Formula \\
\hline Scatter Criteria Matrix [18]. & $S_{k}=\sum_{x \in C_{k}}\left(x-\mu_{k}\right)\left(x-\mu_{k}\right)^{T}$
\end{tabular}

Where, $C_{k}$ is the set of instances in cluster $k ; \mu_{k}$ is the vector mean of cluster $k$.

Condorcet's criterion is another approach applied for the ranking problem [19].

$$
\sum_{C_{i} \in C} \sum_{\substack{X_{j}, X_{k} \in C_{i} \\ X_{j} \neq X_{k}}} S\left(X_{j}, X_{k}\right)+\sum_{C_{i} \in C} \sum_{X_{j} \in C_{i}: X_{k} \nexists C_{i}} d\left(X_{j}, X_{k}\right)
$$

Where $S\left(X_{j}, X_{k}\right)$ and $d\left(X_{j}, X_{k}\right)$ measure the similarity and distance of the vectors $X_{j}$ and $X_{k}$.

The $\mathrm{C}$-criterion is an extension of Condorcet's criterion [19].

$$
\sum_{C_{i} \in C} \sum_{\substack{X_{j}, X_{k} \in C_{i} \\ X_{j} \neq X_{k}}}\left(S\left(X_{j}, X_{k}\right)-\gamma\right)+\sum_{C_{i} \in C} \sum_{X_{j} \in C_{i}: X_{k} \nexists C_{i}}\left(\gamma-S\left(X_{j}, X_{k}\right)\right.
$$

Where $S\left(X_{j}, X_{k}\right)$ and $d\left(X_{j}, X_{k}\right)$ measure the similarity and distance of the vectors $X_{j}$ and $X_{k} ; \gamma$ is the threshold value.

Sum of Square Error (SSE) is the utmost regularly used criterion measure for clustering [19].

$$
S S E=\sum_{k=1}^{K} \sum_{\forall x_{i} \in C_{k}}\left\|x_{i}-\mu_{k}\right\|^{2}
$$

Where $C_{k}$ is the set of instances in cluster $k ; \mu_{k}$ is the vector mean of cluster $k$ 
Table 2. External Quality Criteria Measure with Their Formula

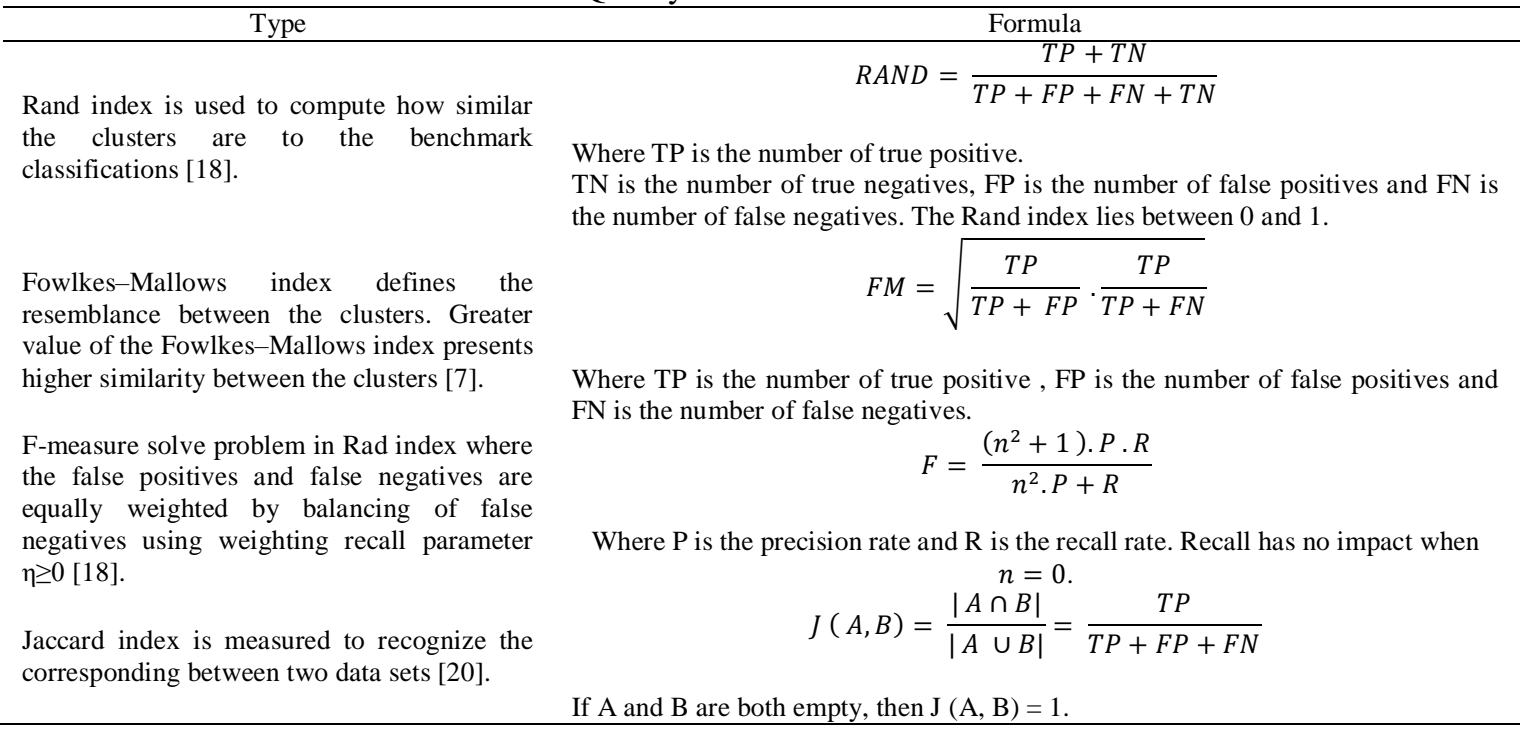

Internal criteria as shown in Table 1 are commonly used to calculate the decisiveness of the clusters by spreading correspondence measure methods. It calculate how closely the objects linked in a cluster by evaluating the cluster compactness based on variance [21]. The external value standards measure as shown in Table 2 can be beneficial in command to match the assembly of cluster to a predefined classification of the occurrences. An external index is made of consideration of prior info on the data created on several prespecified structure and used as an average to legalize the clustering results. On the contrary, the internal tests are not reliant on prior knowledge as it inspects the clustering organization straight as of the original data.

\section{METHODOLOGY}

SNNs models contains of single or additional layers of connected neurons which are analogous to the ANNs structural design such as shown in Figure 2 but contrast in the neuron's model and the type of the activation function [22]. Spiking neurons can be demonstrated in many diverse ways. In order to quantitatively define neuronal performance based on comprehensive modelling of the neuronal membrane potential and ion channel conductance, a number of detailed mathematical or biophysical models have been established. These structures of actual neurons turn out to be a central model of a concept for spiking neural reproductions.

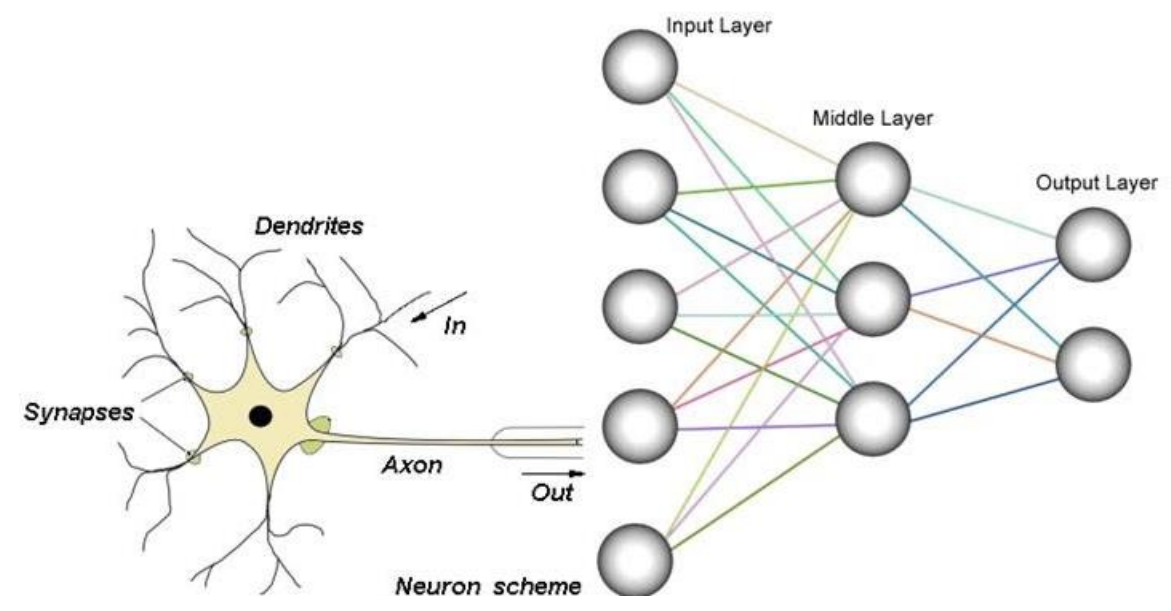

Figure 2. Biological Neurons and Neural Network Neuron Model taken from [23] 
The common properties of all spiking models shared with their biological counterparts based on the conceptual view are as followed: (1) Their possibility of firing (generating a spike) is augmented by excitatory inputs and reduced by inhibitory inputs, (2) their dynamics is regarded as by at least one state variable. The model is thought to produce one or more spikes when the internal variables of the model reach a certain state, and (3) they process info approaching from many inputs and yield single spiking output signals [11].

Therefore, such comprehensive networks are significant for learning the properties of numerous spiking configurations particularly in relations of network simulation formations. The explicitly knowledge on which neurons are stimulated and whether certain neurons are stimulated more often than others are crucial. In simplified way, SNNs are the networks of spiking neurons and SNN construction is habitually comparable to the traditional ANN. Figure 3 show the spiking neural networks with a few inputs, their connections and output obtain as spike compared with the figure of biological neurons connections. Three general categories spiking network topology can be classified into [11]:

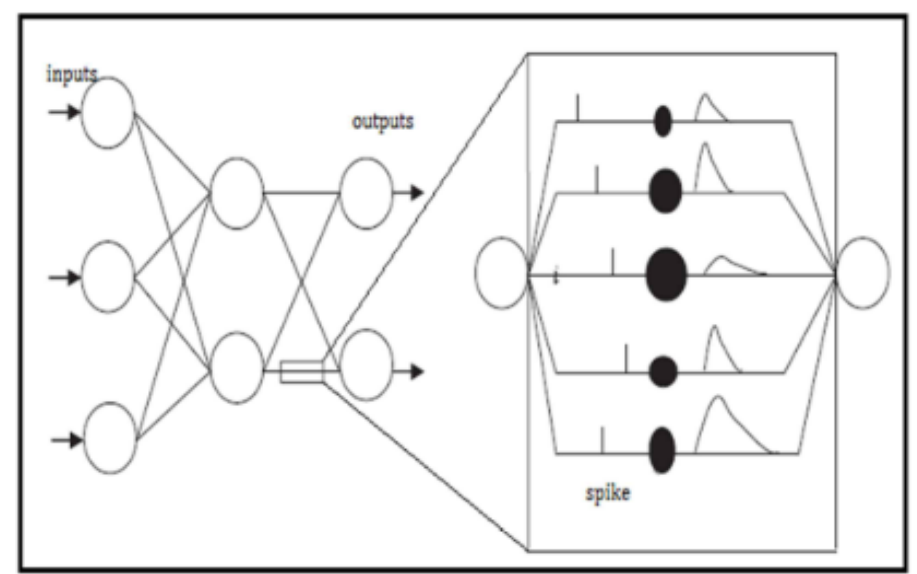

A)

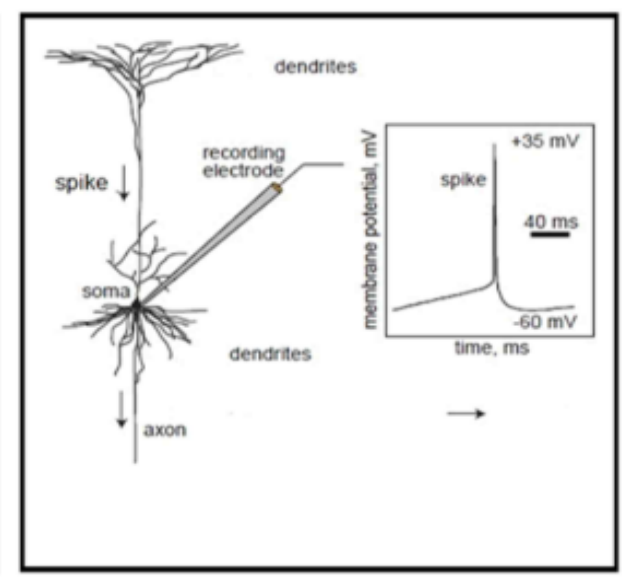

B)

Figure 3. A) Spiking Neural Networks and their connections versus B) Biological neurons taken from [18]

\subsection{Feed Forward Networks}

Originate mainly in areas closer to the periphery in biological neural systems. The data input to output units is firmly flow from one direction here but no feedback connections are present even when the data processing can spread over several layers of neurons as shown in Figure 4.

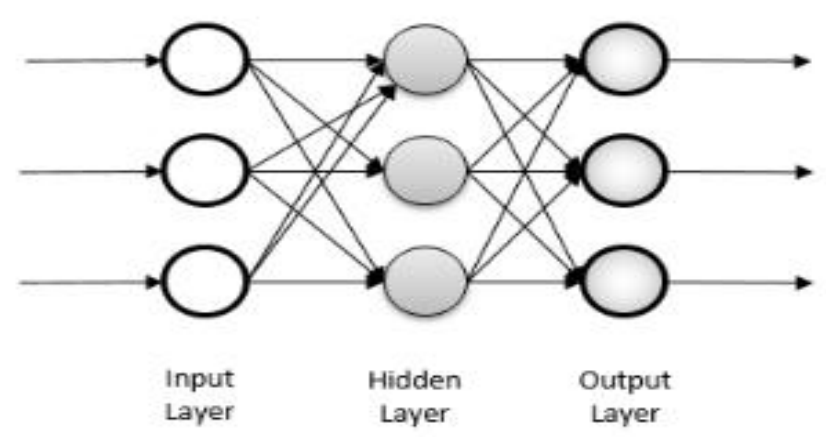

Figure 4. Feed forwards networks architecture taken from [24]

Equally, in SNN feed forward topology are typically useful to exhibiting low-level sensory systems, tactile or sensing olfaction. Feed forward networks are explored correspondingly in the perspective of spike synchronous for resolving the binding problematic grounded on spatial-temporal configurations of spikes. 


\subsection{Recurrent Networks}

At this point single neurons or populations of neurons cooperate over reciprocal (feedback) relations. Feedback relations effect in an inner state of the network which permits it to demonstrate dynamic temporal behavior as shown in Figure 5. Therefore, recurrent networks are reflected by a higher dynamics and theoretically complex computational capabilities compared with feed forward networks resulting in more challenge to control and train.

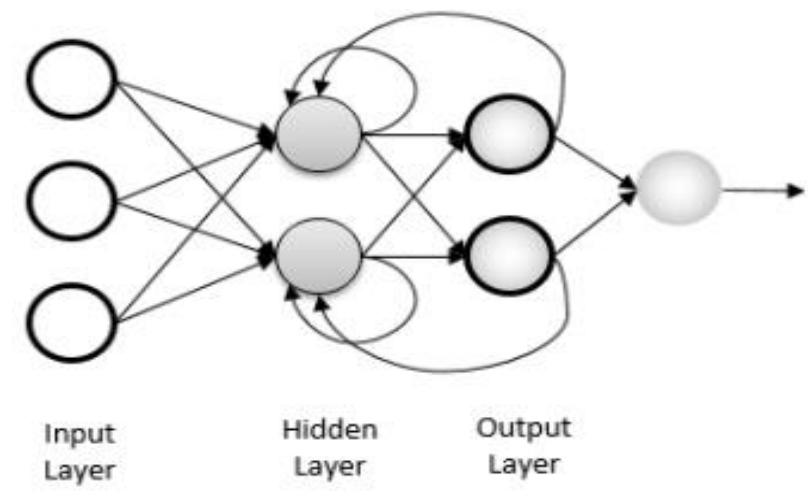

Figure 5. Recurrent networks architecture taken from [24]

In order to investigate neural information processing involved in formation of associative memories or working memory, recurrent spiking neural networks is used. Spiking networks with recurrent relations have also been used to model and examine occurrences observed in the brain that arise from complex dynamics of reciprocal interactions for example network fluctuations or network multi-stability. In the competition between neurons or neural populations or signal decor relation, networks with lateral inhibitory communication is repeatedly used and reflected in the context of decision making.

\subsection{Hybrids Networks}

This group comprehends networks in which selected sub populations might be firmly feed forward, while other has recurrent topology. Communications among the sub populations may be single, directional or mutual.

\section{DISCUSSION}

SNNs depend on the spike timing in their learning and instigation phases which exploit timemissing uninterrupted stimulation functions, compared to the next generation of ANNs. Data clustering via Spiking Neural Networks has been an interest topic to explore recently as this method tends to solve many problems regarding data clustering. In order to used spikes to transfer and study the spatial/spectro temporal data (SSTD) that are programmed with the spiking-time actions for the temporal data and with the location of the synapses for the spatial data, SNNs struggle to impressionist human neurons. Here a few papers that has been reviewed regarding the methods of the clustering.

First, STDP (Spike Timing-Dependent Plasticity) method state that, a synapse is depressing if the presynaptic spike fires after the post synaptic spike or it will be improved if it helps to induce the fire of the post synaptic spike [25]. This method is an improvement of Hebb's novel work as the work does not deliver some earnings for decreasing the synaptic efficiency but only firming up the synapse efficacy when both the presynaptic neuron and post synaptic neuron are vigorous [25]. This learning function guarantees that only those synapses that influence the post synaptic neuron in a moment before it releases a spike are greatly amplified. The information for each input pattern by which interval supplies can be shown by this method. The results demonstrate that this model can do data clustering effectively and can extent a stable status by giving sufficient data testers.

SpikeCD, spiking neural network with clustering degeneracy strategy of a parameter-insensitive method is proposed to improve clustering competence of SNN and increase accuracy by solving problem regarding Rank Order based algorithms where threshold of similarity is need to be predefined for construction alteration which is reflected time cost and be determined by a lot of previous understanding [26]. Thus, the structure adjustment process is tough to be optimized. This method combines supervised 
learning and unsupervised clustering. The number and focal point of spiking RBF neurons are adjusted conferring to input data in hidden layer and are attuned by manipulating the resemblance and variance of training data. The number of outstanding neurons after advancement in this way is much less than the amount of those addition approaches produced by arbitrary correspondence and the predefined parameters. As a consequence, SpikeCD can succeed worthy accuracy with modest assemblies.

Another enhancement of rank order-based learning approach is known as SpikeTemp, for SNNs with adaptive construction. This feed forward trained SNN contains of two layers of spiking neurons: first, an encoding level which temporally converts real-valued structures into spatial-temporal spike configuration. Second, a production level of dynamic grown neurons which accomplish the spatial-temporal classification [27]. This technique engaged both Gaussian approachable fields and square cosine populace training scheme to convert real-valued structures into spatial-temporal spike configurations [27]. SpikeTemp method tried to solve issues regarding the precise timing information which is being ignored and the speed of imitation time of the network for huge data speed and networks. It is appropriate for inputs denoted by a 1-D feature vector and extra proper for online schemes. Consequently, this method is computationally effective and supplementary appropriate for varied collection of data set.

A novel learning procedure for spiking neural networks constructed on revision of the inner neuron threshold is recommended as in [28]. This threshold revision is engaged to support spiking neural neuron to fire the lowest amount of output spike to reserve all material of the input spike train on the equivalent period [28]. The inadequate presentations owed by the usage of single sensors evaluation values nonetheless the period of these evaluations strained to be resolve in this algorithm, but this actual time motion prediction, recognition and behavior mechanization may perhaps need the sensor clarifications time input along with the sensor values. Permitted to clustering and to vary the compound user actions, a decrypt method using SNN appearances is constructed. Then, the smart environment is trained for cluster user actions inside a particular space by exploiting the SNN ideal. This proposed technique is modest in assembly and can accomplish higher result of clustering [28].

Another approach is unsupervised method to learn and early detection of spatial-temporal patterns using spiking neural networks. In the paper, it was stated difficulties of learning and identifying spatialtemporal patterns is encountered upon demonstrating gestures or other human actions [29]. It used axonal conduction delays that depend on a spike-timing dependent plasticity learning regulation which learn training of individual shapes as sets of polychromous neural clusters. Classification is done by a similarity metric between the sets created on by an improved version of the Jaccard index. The method is assessed on a data set of hand-drawn digits. The data consist of time stamps that are captured simultaneously during the drawing. The outcomes demonstrate that the suggested method can effectively study these configurations based on ominously minor amount of training samples [29]. It also can recognize patterns before their accomplishment. The performance of this method is improved than or equivalent with the three other supervised approaches.

Modelling of large-scale spiking neural models is a significant instrument in the mission to recognize brain purpose and afterward generate real-world applications. A spiking neural network simulant locality called HRL Spiking Simulator (HRLSim) is familiarized as an appropriate manner for employment on a cluster of general-purpose graphical processing units (GPGPUs). It deals with reasonable and accessible instrument for scheming, real-time imitation and investigating of across-the-board spiking neural networks [30]. This HRLSim was premeditated for both corresponding central processing unit (CPU) constructions and parallel general-purpose graphical processing unit (GPGPU) cluster computers. GPUs require a huge amount of single-instruction multiple data (SIMD) processors proficient of capably handling massive volumes of data in equivalent. Besides, the total correlated in creating clusters of GPUs is significantly lesser than CPU-based supercomputers [30].

Lastly, an evolving, dynamic clustering of spatial/spectro-temporal data in 3D spiking neural network models (eSNNs) is introduced for cluster job of spatial-temporal data conducted in the Neucube application of spiking neural network design [6]. The records are encrypted as spike trains before it submitted to the models as each spike signify a variation in the variable rate (an event). Through the unsupervised learning, the cluster centers were predefined by the spatial localities of the input records variables in a 3D SNN model. Afterwards, the clusters are developing through the learning in such they are altered nonstop over time imitating the dynamics of the variations in the records. During the supervised learning, clusters characterize the dynamic series of neuron spiking actions in a trained SNN model, detailed for a certain class of data or for a single occurrence [6]. The suggested clustering approaches allowed us to reasonably analyses the EEG data chronicled from dissimilar subject groups. 


\section{CONCLUSION AND FUTURE WORKS}

Spiking Neural Networks is proved as a significant method to solve complex time-dependent configuration recognition complications for the reason of dynamic illustration naturally inherent in spiking neural network. Theoretically show that, SNN has the skill to estimate any continuous functions. It is also shown that Spike-Temp and Spike-CD can classify diverse data set with better-quality of accurateness and a shorter simulation time is need compared to the rank order method [16]. Moreover, the features representation in this method can be recycled in deep neural networks to excerpt enhanced feature structure [15].

STDP methods focused more on the data clustering that encrypts the input data in temporal space whereas result express by HRLSim propose an actual replication instrument to learn very large-scale spiking neural prototypes. Likewise, clustering activity which is based on adaptive threshold of SNN and learning and recognizing spatiotemporal patterns method using SNN both prove that SNN can significantly handle spatial-temporal data type. Outcomes similarly show that regardless of the exact small amount of training samples, the technique has magnificently summarized the descriptive structures of each class with high classification grades.

The significant purpose of clustering method implying SNN has been summarized in this paper can be used for a better understanding of knowledge discovery on complex data type. Future work regarding Spiking Neural Network clustering approach can be explored by employing the real-world data which higher dimensionality and temporal relation problems. Thus, the significance of SNN in solving dynamic, spatial/spectro-temporal data problem can be proven.

\section{ACKNOWLEDGEMENTS}

The authors would like to acknowledge University Tun Hussein Onn Malaysia (UTHM) and Ministry of Higher Education (MOHE) for the support of this research. This research is supported by University Tun Hussein Onn Malaysia (UTHM) under Postgraduate Research grant (GPPS) vot H062 and Ministry of higher education (MOHE) under the fundamental research Grant Scheme (FRGS) vot 1612.

\section{REFERENCES}

[1] A. A. Abusnaina and R. Abdullah, "Spiking Neuron Models: A Review," Int. J. Digit. Content Technol. its Appl., vol. 8, no. 3, pp. 14-21, 2014.

[2] P. H. Lindsay and D. A. Norman, "Neural Information Processing", vol. 3316. Springer International Publishing, 1977.

[3] S. Ghosh-dastidar and A. G. Lichtenstein, "Review Article Spiking Neural Networks," Int. J., vol. 19, no. 4, pp. 295-308, 2009.

[4] A. Tavanaei, M. Ghodrati, S. R. Kheradpisheh, T. Masquelier, and A. Maida, "Deep Learning in Spiking Neural Networks," Neural Networks, vol. 111, pp. 47-63, Mar. 2019.

[5] R. Wang et al., "Review on Mining Data from Multiple Data Sources," Pattern Recognit. Lett., vol. 109, pp. 120-128, Jul. 2018.

[6] M. G. Doborjeh, N. Kasabov, and Z. G. Doborjeh, "Evolving, Dynamic Clustering of Spatio/Spectro-Temporal Data in 3D Spiking Neural Network Models and A Case Study on EEG Data," Evol. Syst., vol. 9, no. 3, pp. 195-211, 2018.

[7] D. Xu and Y. Tian, "A Comprehensive Survey of Clustering Algorithms," Ann. Data Sci., vol. 2, no. 2, pp. 165-193, 2015.

[8] G. Sehgal and D. K. Garg, "Comparison of Various Clustering Algorithms," 2014.

[9] C. Fraley, "How Many Clusters? Which Clustering Method? Answers Via Model-Based Cluster Analysis," Comput. J., vol. 41, no. 8, pp. 578-588, 2005.

[10] J. A. Dickerson, "Biclustering Clustering Gene Expression Data," Bioinformatics, pp. 1-14, 2011.

[11] F. Ponulak and A. Kasiński, "Introduction to Spiking Neural Networks: Information Processing, Learning and Applications," Acta Neurobiol. Exp. (Wars)., vol. 71, no. 4, pp. 409-433, 2011.

[12] R. M. Cesar and L. da Fontoura Costa, "An Introduction to Neural Networks, vol. 14, no. 1. 2002.

[13] M. Othman, "Spatial-Temporal Data Modelling and Processing for Personalised Decision Support," Dr. Diss. Auckl. Univ. Technol., 2015.

[14] N. K. Kasabov, Time-Space, "Spiking Neural Networks and Brain-Inspired Artificial Intelligence", vol. 7. Berlin, Heidelberg: Springer Berlin Heidelberg, 2019.

[15] M. Othman, S. A. Mohamed, M. H. A. Abdullah, M. M. Yusof, and R. Mohamed, "A Framework to Cluster Temporal Data using Personalised Modelling Approach," in Advances in Intelligent Systems and Computing, 2018.

[16] K. Kothari and O. Kale, "Survey of Various Clustering Techniques for Big Data in Data Mining," vol. 1, no. 7, pp. $68-71,2014$

[17] M. Awad and R. Khanna, "Machine Learning and Knowledge Discovery," in Efficient Learning Machines, Berkeley, CA: Apress, 2015, pp. 19-38. 
[18] A. Saxena et al., “A Review of Clustering Techniques and Developments," Neurocomputing, vol. 267, pp. 664-681, 2017.

[19] L. Rokach and O. Maimon, "Clustering Methods, Book Section," Data Min. Knowl. Discov. Handb., pp. 321-352, 2005.

[20] J. Leskovec, A. Rajaraman, and J. D. Ullman, "Mining of Massive Datasets". Cambridge: Cambridge University Press, 2014.

[21] Y. Liu, Z. Li, H. Xiong, X. Gao, and J. Wu, "Understanding of Internal Clustering Validation Measures," Proc. IEEE Int. Conf. Data Mining, ICDM, pp. 911-916, 2010.

[22] M. Awad and R. Khanna, "Deep Neural Networks," in Efficient Learning Machines, Berkeley, CA: Apress, 2015, pp. 127-147.

[23] "Artificial Neural Network | Types | Feed Forward | Feedback | Structure | Perceptron | Machine Learning | Applications | Tech Blogs - MSA Technosoft." [Online]. Available: https://msatechnosoft.in/blog/techblogs/artificial-neural-network-types-feed-forward-feedback-structure-perceptron-machine-learning-applications. [Accessed: 02-Feb-2019].

[24] E. Pekel and S. S. Kara, "A Comprehensive Review for Artifical Neural Network Application to Public Transportation," vol. 35, no. 1, pp. 157-179, 2017.

[25] X. Tao, "Data Clustering Via Spiking Neural Networks Through Spike Timing Dependent Plasticity," Proc. Int. Conf. Artif. Intell. IC-AI 04, 2004.

[26] P. Lin, S. Chang, H. Wang, Q. Huang, and J. He, "Spikecd: A Parameter-Insensitive Spiking Neural Network with Clustering Degeneracy Strategy," Neural Comput. Appl., vol. 6, pp. 1-13, 2018.

[27] J. Wang, A. Belatreche, L. P. Maguire, and T. M. McGinnity, "SpikeTemp: An Enhanced Rank-Order-Based Learning Approach for Spiking Neural Networks with Adaptive Structure," IEEE Trans. Neural Networks Learn. Syst., vol. 28, no. 1, pp. 30-43, 2017.

[28] H. H. Amin, W. Deabes, and K. Bouazza, "Clustering of User Activities based on Adaptive Threshold Spiking Neural Networks,” Int. Conf. Ubiquitous Futur. Networks, ICUFN, pp. 1-6, 2017.

[29] B. Rekabdar, M. Nicolescu, R. Kelley, and M. Nicolescu, "An Unsupervised Approach to Learning and Early Detection of Spatio-Temporal Patterns using Spiking Neural Networks," J. Intell. Robot. Syst. Theory Appl., vol. 80, pp. 83-97, 2015.

[30] K. Minkovich, C. M. Thibeault, M. J. O’Brien, A. Nogin, Y. Cho, and N. Srinivasa, "Hrlsim: A High Performance Spiking Neural Network Simulator for GPGPU Clusters," IEEE Trans. Neural Networks Learn. Syst., vol. 25, no. 2, pp. 316-331, 2014. 